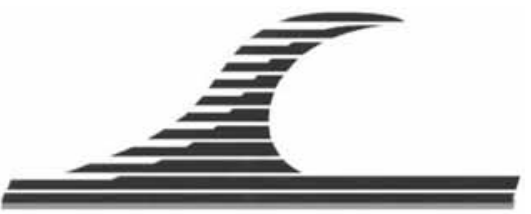

Revue Paralia, Volume 4 (2011) pp 3.1-3.12

Mots-clés : Embouchure tidale, Delta de jusant, Rechargement

de plage, Modèle numérique.

(C) Editions Paralia CFL

\title{
Etude morphodynamique et modélisation numérique de Sebastian Inlet, Floride, USA
}

\author{
Florian G. BREHIN ${ }^{1}$, Gary A. ZARILLO ${ }^{1}$ \\ 1. Department of Marine and Environmental Systems, Florida Institute of Technology, \\ Melbourne, USA. \\ fbrehin@fit.edu
}

\section{Résumé :}

Afin de restaurer les plages en aval de Sebastian Inlet, Floride, USA, des projets de rechargement ont été menés depuis plus de 20 ans, le plus récent datant de 2007. Parallèlement, le district a entrepris une campagne de suivi morphodynamique, comprenant des mesures hydrodynamiques et des relevés bathymétriques biannuels. Ces éléments ont permis à la fois l'analyse de l'évolution des stocks sableux et l'application de modèles numériques couplés du Coastal Modeling System (CMS). Les changements de bathymétrie et du trait de côte sont caractérisés par une forte variabilité spatiotemporelle : une avancée du trait de côte et des dépôts sédimentaires concentrés sur la partie supérieure de la plage sous-marine au sud de la zone de rechargement, ainsi que sur le delta de jusant. Le modèle morphodynamique a permis la reproduction de l'évolution bathymétrique sur l'année 2007, la mise en évidence d'un transport crossshore complexe au niveau des récifs ainsi qu'une inversion dans le transport longshore créé par la réfraction de la houle sur le delta. Ce mécanisme serait à l'origine de l'engraissement du delta de jusant et des plages entre la jetée sud et les barres de swash, remettant en question la localisation des projets de rechargement.

Soumis le 3 novembre 2010, accepté le 16 mars 2011, en ligne le 28 mars 2011.

La seule version examinée est celle écrite en français. La ou les autres versions n'étant pas examinées par le comité de rédaction de la revue, sont donc publiées sous l'entière responsabilité du ou des auteurs. 


\section{2 : Revue Paralia - Vol. 4 (2011)}

\section{Introduction}

Le littoral floridien subit depuis une trentaine d'années une érosion accentuée due à la stabilisation des embouchures tidales par des jetées. Afin de restaurer les plages en aval, de nombreux projets de rechargement ont été mis en place depuis les années 1970, avec des résultats souvent discutables. Des relevés bathymétriques successifs permettent de quantifier les échanges sédimentaires mais la résolution temporelle reste limitée et il est difficile de comprendre les interactions sur des échelles de temps courtes, de l’ordre de quelques heures par exemple lors de tempêtes. Des simulations numériques permettent d'améliorer la compréhension des mécanismes de transport et la réponse morphologique spécifique aux conditions hydrodynamiques. Cet article présente les résultats d'un projet de suivi morphodynamique et de modélisation numérique de l'embouchure tidale de Sebastian. Les calculs de changements de bathymétrie et du trait de côte sont effectués sur plusieurs périodes entre 2000 et 2009, incluant des évènements importants (cyclones et projets de rechargement). Ces résultats sont combinés à des données hydrodynamiques pour appliquer et calibrer un modèle morphologique (couplage CMSFlow/CMS-Wave de l'armée américaine), qui reproduit le transport sédimentaire et les évolutions morphologiques de l'embouchure et des plages adjacentes.

\section{Zone d'étude}

\subsection{Situation géographique et géomorphologique}

Sebastian Inlet se situe sur la côte Est de Floride et sépare la lagune d'Indian River de l'Océan Atlantique (figure 1). L'île barrière aux alentours ne dépasse pas les $2 \mathrm{~km}$ de largeur ni les $10 \mathrm{~m}$ de hauteur au-dessus du niveau moyen. La zone est caractérisée par une grande variabilité dans la taille des sédiments $(0,2$ à $0,5 \mathrm{~mm})$, mais aussi dans leur composition. L'embouchure atteint sa configuration finale dans les années 1970, après le dynamitage dans la roche pléistocène "coquina" d'un chenal étroit et d'une trappe à sable (extrémité Ouest du chenal), et la stabilisation à l'aide de deux jetées (figure 1). La géomorphologie de l'embouchure est marquée par une série de récifs submergés au sud, et un delta de jusant conséquent qui s’est développé à la suite de la stabilisation et se rattache à la côte au sud à 1 km environ (ZARILLO \& BREHIN, 2009).

\subsection{Projets de rechargement}

Les projets de rechargement couvrent environ $3 \mathrm{~km}$ de plage en aval de l'embouchure, entre les marqueurs "R5" et "R16" (figure 1). Ces points de référence utilisés pour les levés de plage, ont été placés par le département de protection environnementale de Floride (FDEP) tous les 300 m et s’incrémentent du nord au sud de chaque comté. Les rechargements totalisent environ $1000000 \mathrm{~m}^{3}$ sur une vingtaine d'années et consistent en un dragage de la trappe à sable ou de sources externes à l'embouchure, et placement du sable via un système de pompes et de pipelines. Le projet "Ambersand" qui remonte 
à 2003 est le plus conséquent $\left(500000 \mathrm{~m}^{3}\right)$. Le plus récent dragage de la trappe date de mars 2007, avec $80000 \mathrm{~m}^{3}$ de sable dragués et placés entre R5 et R15. En novembre 2007, $150000 \mathrm{~m}^{3}$ additionnels provenant d'un site externe seront placés entre R8 et R15, suite à une saison des cyclones intense. Depuis 1990, le district a mis en œuvre un plan de suivi morphodynamique qui inclut des relevés hydrographiques et des photographies aériennes.

\subsection{Climat hydrodynamique}

Une station météorologique est maintenue sur la jetée nord par le laboratoire d'ingénierie côtière du Florida Tech (figure 1) ainsi que des capteurs de type ADCP ancrés dans $6 \mathrm{~m}$ de fond au nord de l'embouchure. Ce système est constitué d'un capteur principal RDI Workhouse Sentinel et d'un capteur secondaire Nortek AquaDopp, déployés sur une durée de 2 à 3 mois. Ces instruments prélèvent des données de houle (hauteur, période et direction) à $2 \mathrm{~Hz}$ pour 1024 échantillons par burst toutes les 3 heures. Les données de courant (vitesse et direction) sont collectées à $24 \mathrm{~Hz}$ pendant 2 minutes toutes les 30 minutes. Ce système a permis de caractériser le climat hydrodynamique sur le long terme et lors de passages de tempêtes, mais également de calibrer et de valider des modèles numériques, avec des comparaisons de courants et hauteurs de houle (ZARILLO \& BREHIN, 2009).

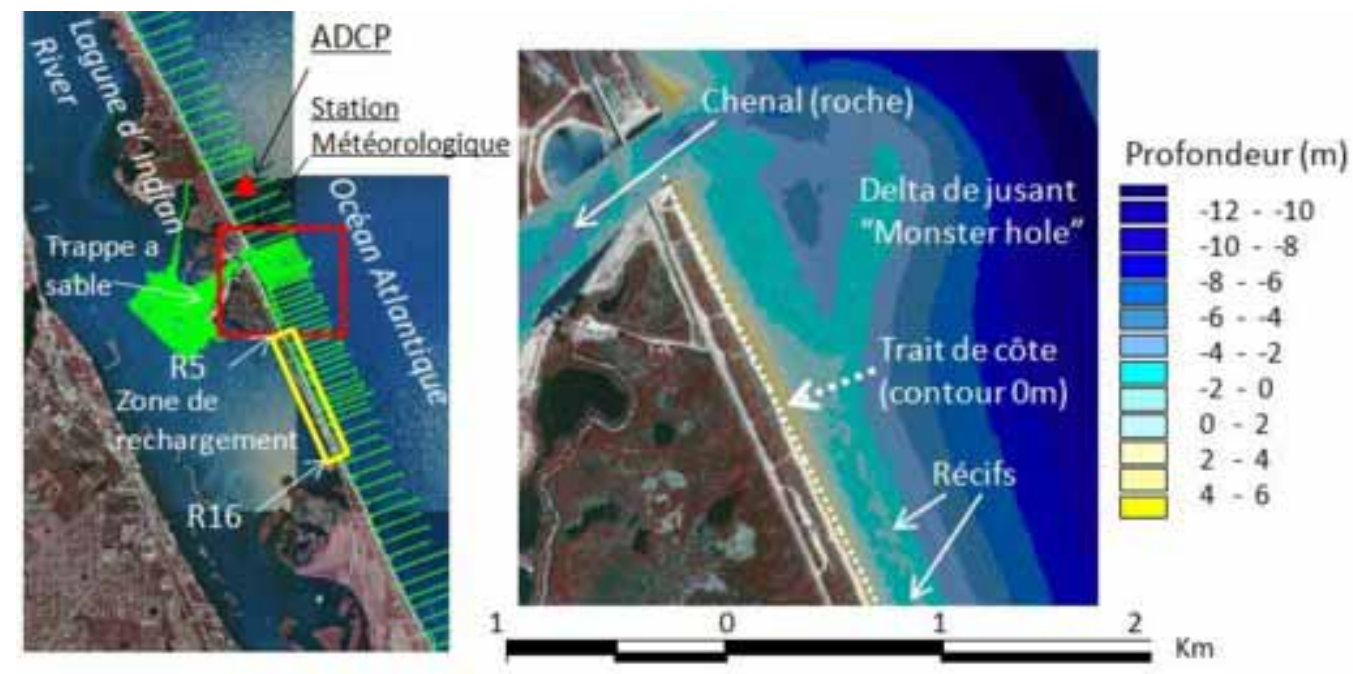

Figure 1. Zone d'étude et couverture des relevés bathymétriques en vert.

L'embouchure est dominée par la houle, avec une hauteur moyenne annuelle de 0,6 m (pouvant dépasser les $3 \mathrm{~m}$ durant les tempêtes hivernales et les cyclones en été), et un fort signal saisonnier. Le transit littoral moyen de direction sud peut atteindre $250000 \mathrm{~m}^{3}$ /an et résulte d'un transport vers le sud en hiver et vers le nord en été (ZARILLO \& BREHIN, 2009). La marée est de type semi-diurne microtidale avec une amplitude océanique d'un peu plus de $1 \mathrm{~m}$, qui diminue fortement dans la lagune. 


\section{4 : Revue Paralia - Vol. 4 (2011)}

La combinaison d'un chenal étroit et le volume important de la lagune (prisme tidal de $10 \times 10^{6} \mathrm{~m}^{3}$ ) génèrent de forts courants de marée (2,5 m/s en vives eaux entre les jetées), qui limitent le dépôt sédimentaire dans le chenal et sont à l'origine de la formation des deltas. Les interactions houle-courant à marée descendante accentuent les risques de navigation et rendent les mesures in situ difficiles.

\section{Matériel et méthodes}

\subsection{Les changements bathymétriques et du trait de côte}

Des données de bathymétrie ont été collectées bi annuellement depuis 1990 par des compagnies locales spécialisées, conformément aux normes du manuel de relevés hydrographiques de l'armée américaine (USACE, 1994). La couverture inclut une zone d'environ $20 \mathrm{~km}$ (figure 1), avec une résolution maximale autour de l'embouchure (variant de 1 à $5 \mathrm{~m}$ selon les années). Les données de latitude, longitude et élévation $(\mathrm{x}, \mathrm{y}, \mathrm{z})$ ayant des coordonnées locales "state plane 1927" (horizontal) et NGVD29 (vertical) en mètres, sont incorporées dans un logiciel de SIG (Arcview GIS3.2). Des modèles numériques de terrain (MNT) sont ensuite générés pour déterminer l'évolution bathymétrique sur une multitude d'échelles de temps et pour extraire le trait de côte à partir du contour des $0 \mathrm{~m}$ hydrographique (figure 1 ).

\subsection{Description et application des modèles numériques}

Le modèle morphodynamique repose sur un couplage du modèle de propagation de houle (CMS-Wave) et du modèle d'écoulement de marée (CMS-Flow), appartenant au Coastal Modeling System (CMS) développé par le Laboratoire d'hydraulique côtière de l'armée américaine. Le modèle hydrodynamique bi-dimensionnel horizontal (ancien code M2D) utilise une méthode des volumes finis et possède un code de calcul de transport sédimentaire avec la possibilité de simuler les zones non érosives (BUTTOLPH et al., 2006). Le modèle de houle (ancien WABED), est un modèle de propagation des vagues de phase moyenne et inclut des phénomènes tels que réfraction, diffraction, réflexion, ainsi que les interactions houle-courant (DEMIRBILEK et al., 2007). Le couplage s'effectue sur l'année 2007 toutes les trois heures à l'aide du "Steering Module" de l'interface SMS (Surface Water Modeling System). Les grilles de modélisation s'étendent sur environ $10 \mathrm{~km}$ de part et d'autres de l'embouchure et $5 \mathrm{~km}$ au large (20 m de profondeur). La grille du modèle d'écoulement de marée s'emboîte dans celle du modèle de houle, avec une taille similaire des mailles qui varie entre $50 \mathrm{~m}$ au bord et $200 \mathrm{~m}$ au large. Le trait de côte est digitalisé à partir d'une image aérienne de 2007 et la bathymétrie combine les relevés de janvier 2007 discutés dans la section précédente, et celles du Coastal Relief Model (National Geodetic Data Center). Les zones non érosives au sud de l'embouchure provenant d'une campagne récente de caractérisation des fonds sous marins à l'aide d'une sonde acoustique mono faisceau 
(type RoxAnn) sont inclues dans la grille, ainsi que des stations d'observation pour l'extraction du transit littoral (total de 62 rangées de 5 cellules sur l'ensemble du domaine représentant la plage sous-marine jusqu'à une profondeur d'environ $3 \mathrm{~m}$ ). Pour le transport sédimentaire, la formule de transport de Lund-CIRP est utilisée, avec une taille moyenne de grain variant entre $0,2 \mathrm{~mm}$ et $0,3 \mathrm{~mm}$. Pour le modèle hydrodynamique, les conditions aux limites sont issues du logiciel de prédiction de marée IOS. Des séries de hauteur d'eau extraites toutes les heures sont appliquées aux extrémités est (océan), nord lagune et sud lagune de la grille du modèle. La vitesse et la direction du vent provenant de la station météorologique sont appliquées uniformément à la grille. Les conditions aux limites du modèle de houle (hauteurs, périodes et directions de pic) proviennent d'une simulation à plus grande échelle du même model CMS-Wave forcé avec les données de Wavewatch 3 (TOLMAN, 2009).

\section{Résultats et discussion}

\subsection{Evolution bathymétrique et du trait de côte}

Les changements bathymétriques sur le long terme (figure 2, en haut à gauche) indiquent un fort engraissement des deltas, de la trappe à sable, et de la plage directement en aval de l'embouchure entre la jetée sud et les barres de swash (jusqu'à $+2 \mathrm{~m}$ ). Ces changements correspondent à des gains volumiques totalisant environ $+180000 \mathrm{~m}^{3}$ pour l'ensemble de ces zones. La section de plage de R1 a R3 subit une forte avancée du trait de côte (+35 m) durant cette période (figure 3a), alors que la zone de rechargement (R5 à R15) est caractérisée par un recul (-10 à -25 m), et l'érosion généralisée de la partie supérieure de la plage sous-marine $\left(-200000 \mathrm{~m}^{3}\right)$. Des zones de déposition atteignant $+30000 \mathrm{~m}^{3}$ sont observées sur les plages au sud de la zone de rechargement (R20-R30).

L'évolution bathymétrique de 2003 à 2007 (figure 2, en haut à droite) montre des similarités avec la période précédente : une érosion importante $\left(-150000 \mathrm{~m}^{3}\right)$ de l'avant plage de la zone de rechargement (R5-R15), compensée par des zones de déposition de $+50000 \mathrm{~m}^{3}$ et $+75000 \mathrm{~m}^{3}$ respectivement sur la section de plage directement en aval de l'embouchure (R1-R5) et au sud de la limite des projets de rechargement (R15-R30). Ces résultats suggèrent un transport sédimentaire à la fois vers l'embouchure et vers le sud (interactions avec les récifs). L’évolution du trait de côte pour cette période (figure 3b) souligne une avancée au sud de la limite du projet (+25 m), suggérant que le rechargement de 2003 a été bénéfique à la zone en aval. 

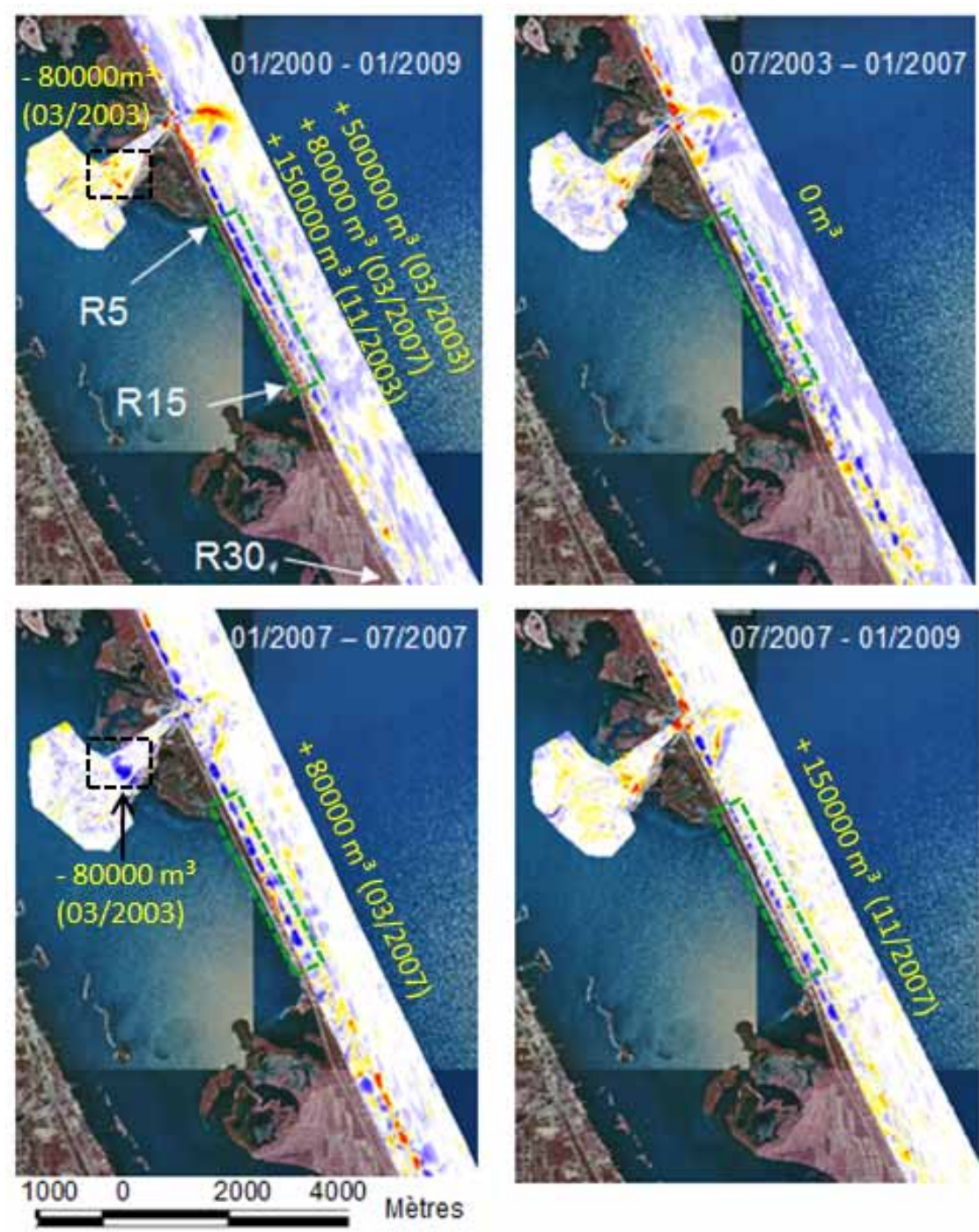

\section{Changement bathymétrique $(\mathrm{m})$}

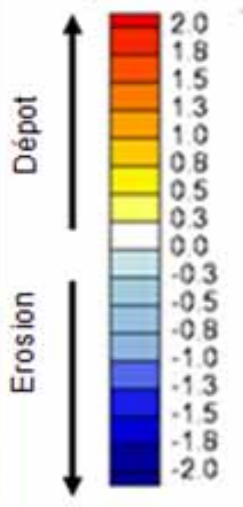

Figure 2. Changements bathymétriques pour plusieurs périodes entre 2000 et 2009. Les volumes correspondent aux quantités de sable draguées et rechargées.

Les différences bathymétriques entre janvier et juillet 2007 (figure 2, en bas à gauche) illustrent l'érosion de la trappe à sable résultant des opérations de dragage $\left(-80000 \mathrm{~m}^{3}\right)$, et de la partie supérieure de la plage sous-marine située entre la jetée sud et R15 $\left(-180000 \mathrm{~m}^{3}\right)$. Les dépôts sédimentaires sont concentrés sur la partie inférieure de la plage sous-marine de la zone de rechargement (R4-R16) et au sud de R20, totalisant respectivement $+30000 \mathrm{~m}^{3}$ et $+80000 \mathrm{~m}^{3}$. Ces observations, combinées à l'absence de dépôt significatif sur le delta de jusant, suggèrent un transport important vers le sud qui serait dû en partie au cyclone Andrea (mai 2007). L’évolution du trait de côte (figure 3c) sur cette période remet en question l'efficacité du projet de mars 2007, avec des avancées concentrées sur la section de plage de la jetée sud à R4 (+50 m) et au sud de R20 (+20 m) alors que la section rechargée subit un recul de 10 m en moyenne. 
Les changements de juillet 2007 à janvier 2009 (figure 2, en bas à droite) indiquent l'érosion de la partie supérieure de la plage sous-marine de la section rechargée $\left(-35000 \mathrm{~m}^{3}\right)$ et les dépôts au pied des jetées, et au sud de la zone de rechargement (respectivement $+15000 \mathrm{~m}^{3}$ et $+60000 \mathrm{~m}^{3}$ ). Le trait de côte (figure 3d) avance faiblement sur la partie rechargée (+5 à +10 m) et recule au sud de R15, entraînant une partie des dépôts observés sur le bas de plage de cette zone.

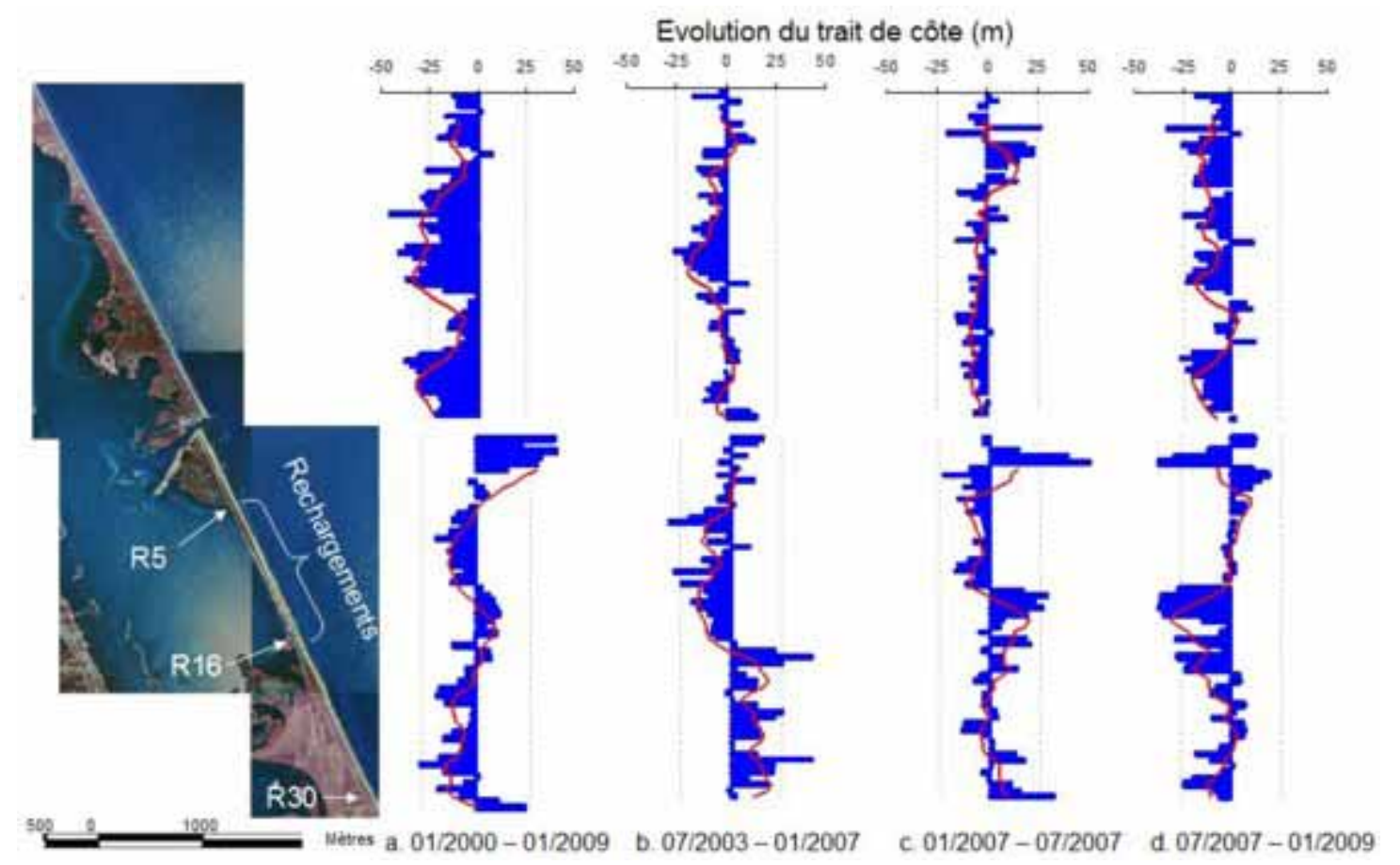

Figure 3. Changements du trait de côte sur plusieurs périodes entre 2000 et 2009.

\subsection{Modélisation morphodynamique}

L’évolution bathymétrique reproduite par le modèle sur l'année 2007 (figure 4, gauche) indique un fort dépôt sur le delta de jusant (+2 m), la partie supérieure de la plage sous marine, et l'extrémité Est du chenal. L'érosion domine la partie ouest du chenal, la plage inférieure et la bordure du delta. Le modèle compare l'évolution morphologique en fonction du climat hydrodynamique. Deux évènements importants interviennent en 2007. Le cyclone Dean, circulant dans les Caraïbes, génère une houle moyenne d'orientation SE $\left(\mathrm{H}_{\mathrm{s}}=1,2 \mathrm{~m}, \mathrm{~T}_{\mathrm{p}}=13 \mathrm{~s}\right)$, représentative des conditions peu énergétiques estivales. Le cyclone Andrea longe la côte floridienne en générant une forte houle d'est/nord est $\left(\mathrm{H}_{\mathrm{s}}=3 \mathrm{~m}, \mathrm{~T}_{\mathrm{p}}=15 \mathrm{~s}\right)$, similaire aux conditions des tempêtes hivernales. 


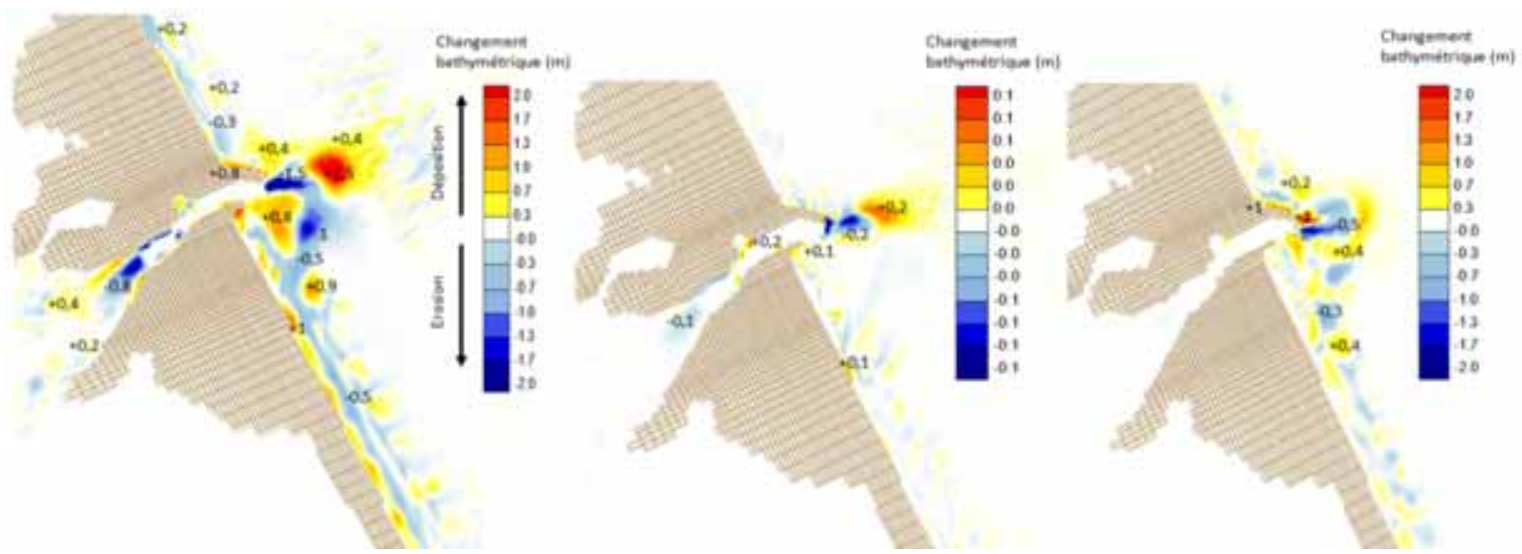

Figure 4. Changements bathymétriques calculés par le modèle : Année 2007 (gauche), cyclone Dean, 72 heures (milieu), cyclone Andrea, 72 heures (droite).

Les changements bathymétriques modélisés pour le cyclone Dean (figure 4, milieu) montrent une faible érosion (-0,1 m) à l'extrémité de la jetée nord, un dépôt sur la partie offshore du delta $(+0,1 \mathrm{~m})$, et des changements peu significatifs sur le haut de la plage sous-marine au sud. Le transit littoral (figure 5, milieu) est dirigé vers le nord et de faible intensité (maximum de $+250 \mathrm{~m}^{3}$ ), ce qui est en accord avec l'orientation de la houle.
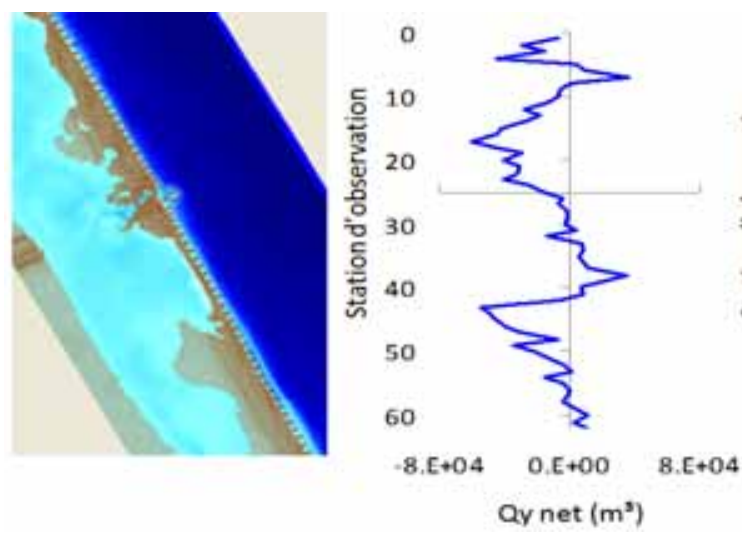
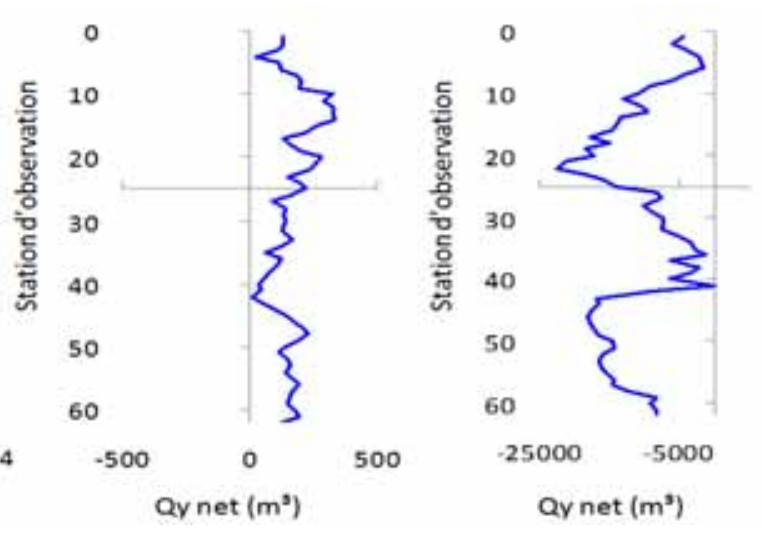

Figure 5. Transport littoral calculé par le modèle : Année 2007 (gauche), cyclone Dean, 72 heures (milieu), cyclone Andrea, 72 heures (droite).

Les changements dus au cyclone Andrea (figure 4, droite) sont beaucoup plus importants, avec des dépôts sédimentaires le long de la jetée nord et sur le delta de jusant (+1 m), ainsi qu'une alternance entre des zones de dépôt et d'érosion sur la partie supérieure de la plage sous-marine au sud de l'embouchure. Le transit littoral vers le sud atteint presque $20000 \mathrm{~m}^{3}$ sur certaines zones (figure 5, droite). Sur le long terme, le transport est majoritairement dirigé vers le sud (valeurs négatives). Les résultats indiquent toutefois des variations spatiales complexes : une réduction de l'intensité du transport près de l'embouchure et une inversion dans la direction entre l'embouchure et 
la station d'observation 50 correspondant au monument R16 (limite sud de la zone de rechargement). Sur la zone en aval (R16-R30), l'influence de l'embouchure (jetées et delta de jusant) sur le transport sédimentaire et la dérive littorale est négligeable d'où l'augmentation dans l'intensité du transport vers le sud. Il est également important de noter que cette zone est caractérisée par une variation dans la distribution des zones de récifs (figure 6). L’observation de la couverture et des coupes longitudinales pour 3 stations (R5, R15 et R25) montre que ces récifs se situent plus loin du rivage au sud de R16, et sont marqués par un relief plus accentué. Cette configuration particulière favorise l'accumulation du sable provenant de la zone rechargée et transporté par la dérive littorale.
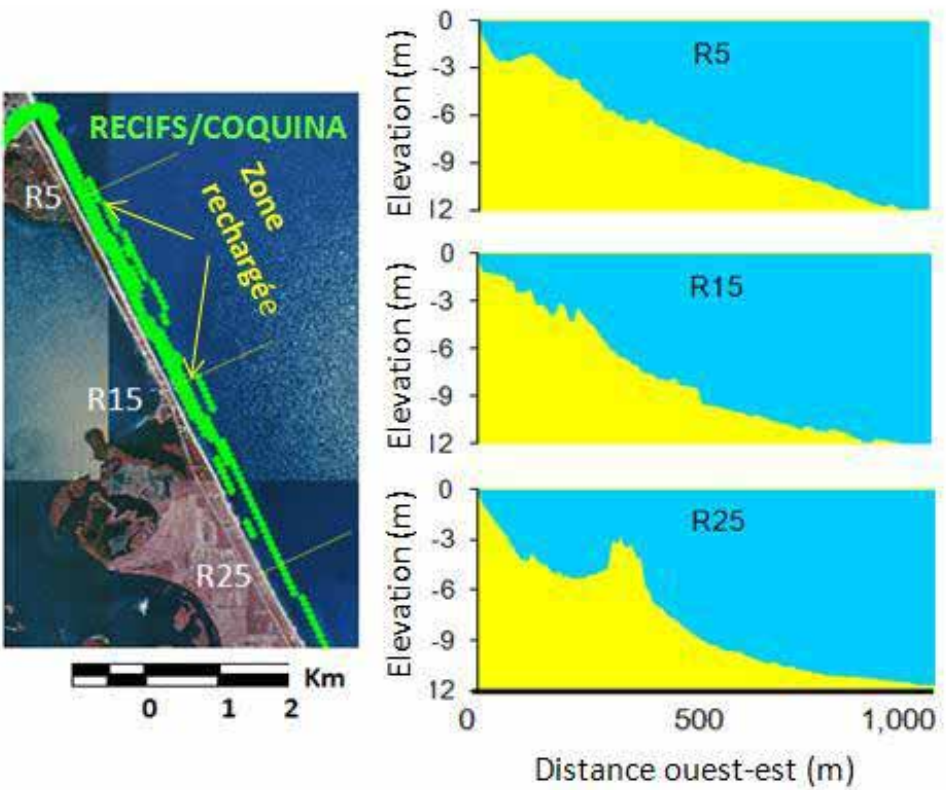

Figure 6. Location des zones de récifs et coupes transversales pour 3 stations (bathymétrie de Juillet 2009).

Les champs de hauteurs de houle calculés par le modèle pour le cyclone Andrea illustrent clairement l'augmentation de la hauteur significative et le phénomène de réfraction aux abords du delta de jusant (figure 7). Ce phénomène de réfraction serait à l'origine de l'inversion dans la direction du transport sédimentaire entre R1 et R4 durant les houles de tempêtes (figure 8). Les simulations montrent que l'inversion dans le transport (ensablement de l'embouchure) semble être favorisée durant le flot, tandis que le mécanisme de by-pass naturel de sédiment (vers le sud) serait favorisé durant le jusant. 

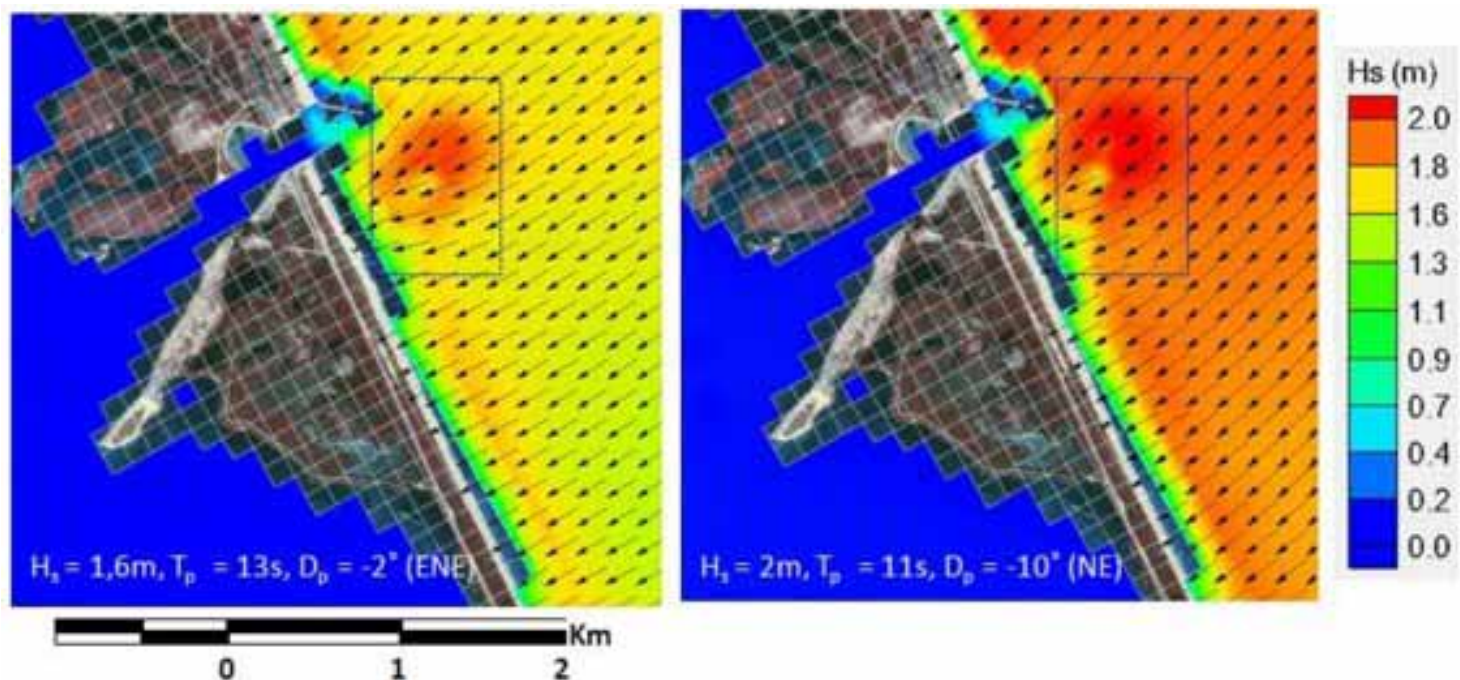

Figure 7. Champs de houle calculés par le modèle (CMS-Wave) durant le cyclone Andrea.
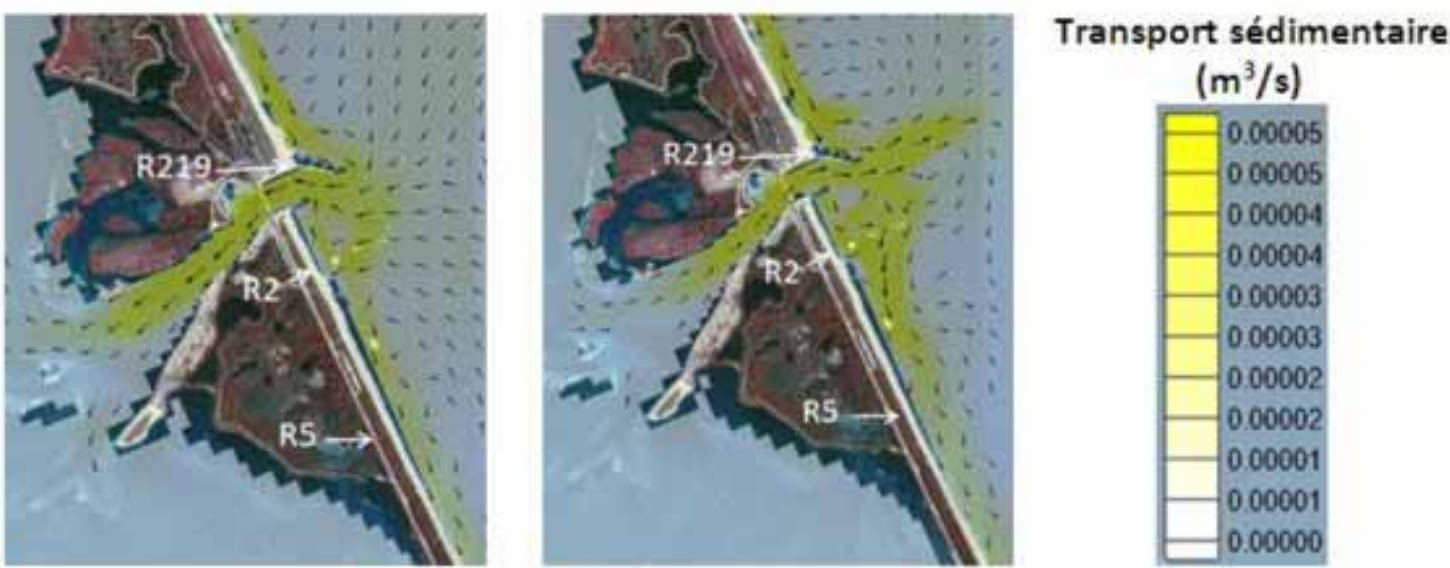

Figure 8. Transport sédimentaire modélisé (CMS-Flow) durant le cyclone Andrea : flot (gauche) et jusant (droite).

\section{Conclusions et perspectives}

Les changements bathymétriques et du trait de côte autour de Sebastian Inlet sur la dernière décennie ont mis en évidence des différences spatio-temporelles complexes et permis la distinction de 3 zones en aval de l'embouchure : de la jetée sud à R5 (influence directe du delta de jusant); de R5 à R16 (zone de rechargement); et de R16 à R30 (complexité dans la distribution des zones de récifs). Les résultats soulignent le contraste du bilan sédimentaire et les variations selon la période étudiée, ainsi que l’importance de la géomorphologie locale (delta, récifs) dans les processus de sédimentation et le contrôle naturel de l'érosion en aval de l'embouchure. Les projets de rechargement de 2003 et 2007 sur la zone R5-R16 (totalisant 730000 m³) $^{3}$ ont eu 
tendance à engraisser les plages situées derrière le delta de jusant (R1-R3) et au sud de la limite des projets (R15-R30). Entre 2000 et 2009, ces zones ont connu des gains volumiques (respectivement $+30000 \mathrm{~m}^{3}$ et $+80000 \mathrm{~m}^{3}$ ), alors que la zone rechargée a perdu environ -200000 $\mathrm{m}^{3}$ de sable. Les simulations numériques du CMS pour l'année 2007 démontrent un comportement correct du modèle sur le plan de l'hydrodynamique et du transport sédimentaire. Les zones d'engraissement et d'érosion sont en accord avec les mesures, même si on note toutefois une tendance à la surestimation des changements bathymétriques modélisés. Les simulations ont également permis de mettre en avant le rôle prépondérant du delta de jusant qui favorise la réfraction de la houle et l'inversion de la direction du transit littoral sur la zone R1-R3. Notre travail consistera à calibrer le modèle morphodynamique sur des échelles de temps plus longues, et à déterminer sa "performance" grâce a une approche probabiliste (score de Brier).

\section{Références bibliographiques}

BUTTOLPH A.M., REED C.W., KRAUS N.C., ONO N., LARSON M., CAMENEN B., HANSON H., WAMSLEY T., ZUNDEL A.K. (2006). Two-dimensional depthaveraged circulation model CMS-M2D: Version 3, Report 2, Sediment transport and morphology change. ERDC/CHL TR-06-09, U.S.A E.R.D.C., Vicksburg, MS.

DEMIRBILEK Z., LIN L., ZUNDEL A. (2007). WABED model in the SMS: Part II Graphical interface. ERDC/CHL. CHETN-I-74. U.S.A E.R.D.C., Vicksburg, MS.

TOLMAN H.L. (2009). User manual and system documentation of WAVEWATCH III version 3.14. NOAA / NWS / NCEP / MMAB Technical Note 276, 194 p.

USACE -U.S. Army Corps of Engineers- (1994). Engineering Manual for Hydrographic Surveys. Washington, D.C., EM 1110-2-103.

ZARILLO G.A, BREHIN F.G. (2009). State of Sebastian Inlet Report: An Assessment of Inlet Morphologic Processes, Historical Shoreline Changes, Local Sediment Budget and Beach Fill Performance.Technical Report 2009-1, Sebastian Inlet District, FL. 
3.12 : Revue Paralia - Vol. 4 (2011) 